\title{
Dual-stage SqueezExpNet: a Deep Neural Network for Facial Expression Recognition
}

This paper was downloaded from TechRxiv (https://www.techrxiv.org).

\section{LICENSE}

CC BY-NC-SA 4.0

SUBMISSION DATE / POSTED DATE

17-02-2022 / 03-03-2022

CITATION

Shahid, Ali Raza (2022): Dual-stage SqueezExpNet: a Deep Neural Network for Facial Expression Recognition. TechRxiv. Preprint. https://doi.org/10.36227/techrxiv.19188902.v1

$\mathrm{DOI}$

10.36227/techrxiv.19188902.v1 


\title{
Dual-stage SqueezExpNet: a Deep Neural Network for Facial Expression Recognition
}

\author{
Ali Raza Shahid ${ }^{1,2}$ and Hong Yan ${ }^{1}$ \\ ${ }^{1}$ Department of Electrical Engineering, City University of Hong Kong, Tat Che \\ Ave, Kowloon, Hong Kong \\ ${ }^{2}$ COMSATS University Islamabad, Park Road, Tarlai Kalan, Islamabad, Pakistan \\ Corresponding author: Ali Raza Shahid (e-mail: a.raza@my.cityu.edu.hk).
}

\begin{abstract}
Facial expression recognition using deep neural networks (DNN) is increasingly important as applications in visual recognition rapidly evolve. Despite efforts to improve the accuracy of FER using DNN, existing methods are not sufficiently generalizable in practical applications, with traditional FER studies mostly limited to controlled conditions. To address controlled and uncontrolled conditions of illumination, pose and occlusion in FER problems, this work proposes a two-fold approach. A model is proposed for variable poses in a controlled situation and for uncontrolled illumination, poses and occlusions in a "wild" FER datasets. A dual stage model, SqueezExpNet, that preserves the attributes of facial features in terms of spatial and geometric regions, followed by a recurrent input classifier, is proposed. SqueezExpNet offers better results as 3D face points are used as input to the network rather than facial regions, which are less important. Analysis and evaluation on three publicly available datasets that cover subject dependent and cross-database tasks, shows SqueezExpNet outperforms existing methods.
\end{abstract}

Keywords: Facial expression recognition, deep convolutional neural network, basic expressions, compound expressions.

\section{Introduction}

Facial expressions are universal signals that can show our emotional state or whether we consent and are a major part of nonverbal communication. Expressions speak louder than words, and about $65 \%$ of information is delivered by the nonverbal communication [1]. Ekman and Friesen [2] defined a coding system based on the movement of one or more facial muscles 
activate an expression. Their facial action coding system (FACS) is used to characterize a set of action units (AU's). The status of these muscles determines the facial expression, which is traditionally encoded into six basic forms (joy, sadness, anger, surprise, fear, disgust) and a neutral state [2]. For example, AU 25 defines the enlargement of two muscles resulting in the lips apart which is typically observed in joy, surprise and fear.

Rapid advances in computer vision and deep learning, have led to significant progress in automatic facial expression recognition systems (FER). However, many of these are restricted to laboratory-controlled environments with posed expressions, uniform illumination, and frontal face views. In practical situations, such as customer feedback, surveillance, autonomous driving, classroom monitoring, and medical treatment, images are obtained in an uncontrolled environment [3].

FER consists of three main steps: image acquisition, feature extraction and classification. Feature extraction aims to maximize interclass variability while minimizing intraclass similarity. Algorithms for FER can be classified into traditional machine learning algorithms and deep learning algorithms. Machine learning algorithms, such as local Gabor features [4], textured descriptors [5] and tensor learning [6], use handcrafted features and perform well in controlled environments, but less so in uncontrolled conditions.

DNN's are widely applied in computer vision problems, such as saliency detection [7], face recognition [8], document image analysis [9], medical image analysis [10], and has been applied to FER [11]. The availability of large datasets, transfer learning of weights and current computational resources, enables DNN's to extract more discriminating features to train a classifier than traditional engineered approaches. A major restriction of facial expression DNN's is that, while they extract spatial relations from input data, they ignore the geometry of facial muscles.

We have investigated human perception and automatic recognition of basic and compound facial expressions in controlled and uncontrolled environments to address real-world illumination levels, variations in pose and occlusion of views. A method which extracts geometrical facial relationships using 3D face points is used to augment spatial data on local facial regions to investigate illumination, poses and occlusion. A dual-stage SqueezExpNet is proposed as an ensemble method that uses 3D geometrical and spatial facial information as recurrent inputs for classification. The incorporation of facial points improves the recognition of facial expressions and prevents the deep convolutional neural network from over-fitting. SqueezExpNet was evaluated on major facial expression datasets (CFEE [12], RaFD [13], and RAF-DB [14]). 


\section{Related Work}

FER systems are usually composed of three main modules, face acquisition, facial feature extraction, and facial expression recognition and the approaches used in each of these steps are surveyed in [15]. For face acquisition, an automatic face detector locates faces in complex scenes, which are then cropped using geometric transformations to localize faces into a unified template [16]. Various methods have been designed to capture facial features and can be categorized into two main groups: texture-based methods and geometry-based methods. Texture-based methods include histogram-oriented gradients [17], local binary pattern [18], and Gabor wavelet coefficients [19], while geometry-based features mainly use facial points around the nose, mouth, and eyes [20], [21]. Combining two or more of these features as a hybrid feature extraction method can improve the representation. Feature classification commonly uses methods such as support vector machines, decision trees, nearest neighbors, and linear discriminant analysis on these classifiers [22], [23], [24].

Deep convolutional neural networks (DCNN) are now widely used in visual computing and pattern recognition tasks. AlexNet [25] introduced this approach, using 61 million parameters and including a dropout layer to avoid overfitting. The VGG-Net family [26] replaced the $3 \times 3$ convolution layer concept with a $7 \times 7$ filter receptive field among the deep networks. However, the size of the model makes it unsuitable for real-time visual applications. Using $3 \times 3$ and $1 \times 1$ convolutional filters along with skipping of connections the ResNet family [27] reduced the depth of the network while maintaining competitive performance.

Neural network design has used deeper and larger models for better classification accuracy regardless of their memory and power requirements in part in the hope that hardware advances will allow these networks to run with real-time performance in embedded systems. However, efforts are being made to reduce the size of the model architecture without compromising accuracy. SqueezeNet [28] archives similar accuracy to AlexNet with $0.2 \%$ the depth of the network and $2 \%$ of the model parameters and this architecture has been successfully used in segmentation and 3D detection [29]. Different Inception Network family layers have been combined with ResNet to reduce the training time [30]. Recurrent Neural Networks (RNN) are also performed for emotion recognition using spatial-temporal information [31]. A hybrid network for facial emotion recognition has be proposed to handle the constraints of spatial images using a CNN-RNN design [32]. 
For practical applications, existing DCNN methods are not sufficiently generalizable. The performance of texture based DCNNs degrade if there are large changes in scenarios [33] and ordinary DCNNs extract spatial information but ignore geometrical characteristics. To overcome this, 3D face points were incorporated into the model using an element by element multiplication of points, masked by weights from the input image. SqueezExpNet, an ensemble of the SqueezeNet [28] type of architecture, preserves spatial information and 3D facial geometrical facial features to emphasize facial components during the training process.

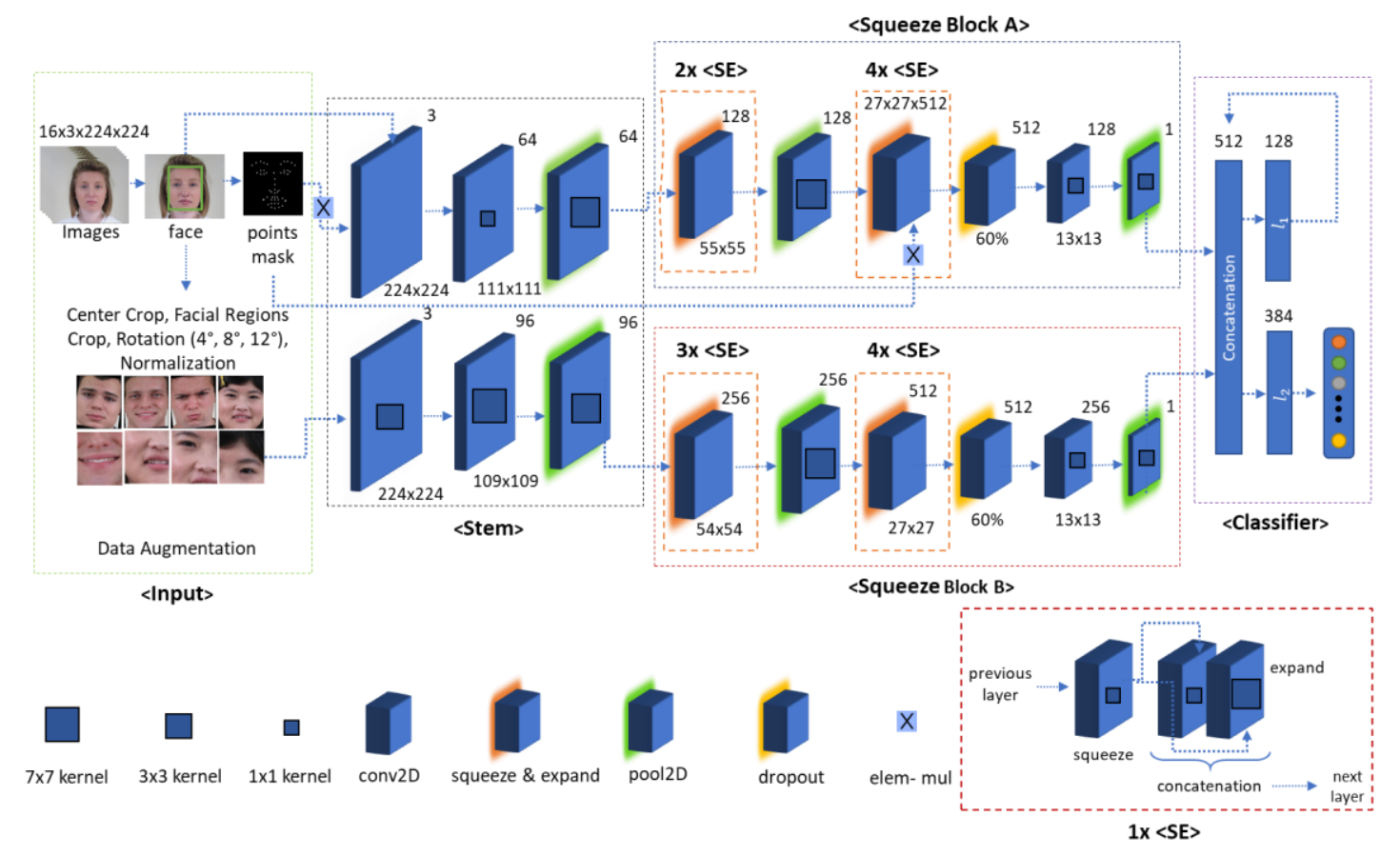

Figure 1. Overview of the proposed methodology. (best viewed in color)

\section{Facial Expression Recognition System}

\subsection{Overview}

Squeezenet based methods, while successful in visual systems [34, 35], do not extract the geometrical relations within the input data. We propose a 3D SqueezExpNet architecture to extract texture and geometry as facial feature maps by an ensemble end-to-end neural network. The geometry component incorporates 3D facial points in the network to achieve more accurate recognition. The last component of the method utilizes a recurrent stacked input unit to assemble enhanced feature maps from Squeeze blocks with final classification of facial expression by a fully connected layer associated with a softmax function.

\subsection{Input}


Detection of faces and facial points: Denote a face image as $I$, its duplicates as $F_{0}, P_{0}$ and crops as face $F_{1}, F_{2}, \ldots, F_{k}$ along face points $P_{1}, P_{2}, \ldots, P_{m}$ with the backbone CNN as $f(x, y)$. The facial feature set $X$ of face $F$ and face point $P$ is defined by:

$$
\begin{gathered}
X=\left[I_{0}, I_{1}, \ldots, I_{k}\right], \\
X=\left[f\left(F_{0} ; P_{0}\right), f\left(F_{1} ; P_{1}\right), f\left(F_{k} ; P_{m}\right)\right]
\end{gathered}
$$

where, $k$ and $m$ are the number of face and face points. As dimensions of images input to a DCNNs are usually fixed, facial images are cropped, and 2D/3D face points extracted using a face alignment method [36]. Then the tensor of the batch (16) of faces in the input is rescaled to $16 \times 3 \times 224 \times 224$ using bicubic interpolation for 3 number of channels as a rectilinear grid. The input tensor is normalized to its mean and standard deviation to standard input to the network. The sequence of means for each channel is $\mu=[0.485,0.456,0.406]$ and standard deviations is $\sigma=[0.229,0.224,0.225]$ is set after number of different trials. Moreover, center crop, facial regions crop transforms are applied to obtain the augmented data for our network. These transforms are applied to augment the data as different facial expressions are mainly defined by different local facial regions. A constraint on cropping within the face (Figure 1) enforces that weights are from local regional areas within the face i.e., mouth, eyes and nose. Rotations from different angles $[-12,-8,-4,0,4,8,12]$ are applied. 2D/3D face points are utilized to construct a face point mask described below.

\subsection{Face Points}

Face points are used to differentiate the importance of the main facial components e.g., mouth, eyes and nose from other parts of the face that are less expressive. A similar approach [37] used different shapes around the face through a binary mask for the CNN to recognize AU's as feature extraction followed by Long-Short Term Memory. Here the various poses are preserved using 3D face points of texture and geometry, followed by recurrent input in an end to end network. The 2D/3D face points are incorporated by placing the element-wise multiplicative layer unit in the stem and Squeeze blocks by multiplying face points by the input tensor (Figure 2). 


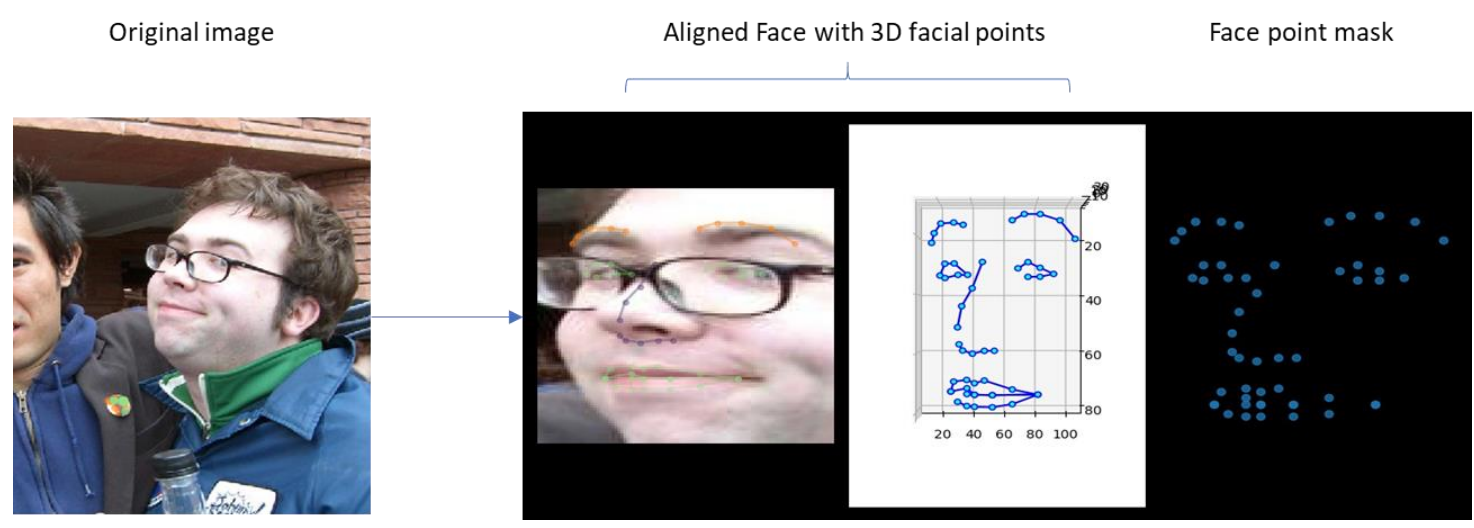

Figure 2 (left) Fifty-one landmarks detected from a facial image and (right) the 2D face mask.

Face points are extracted using OpenCV, a face detection neural network with high accuracy in real-time to obtain the bounding boxes of faces by the caffae model, at a confidence level $>70 \%$. A face alignment method, 2D-3D-FAN is used to localize the face points via a residual block where a DCCN converts 2D annotations to 3D and creates an LS3D-W dataset of 3D face points with $230 \mathrm{~K}$ images [36]. 2D-3D-FAN is used here to extract fifty-one 2D/3D face points from face images with heavy poses, poor illumination and occlusions.

Face points filters are generated for each face image during the training phase. The face points for each face image are resized to their filter size in the stem block of the network. We used to extract fifty-one face points determine the dimensions of the face geometry feature as, $P_{m}$, $m_{i j}=\{1, \ldots, 51\}, x_{i}, x_{j}$, where $i \neq j, X\left(P_{m}\right)=\left(x_{i 1}, x_{j 1}\right)^{T}, x_{i 1}$ and $x_{j 1}$ are the vertical and horizontal components, respectively, of the face points, and their relative positions are $r_{P}=$ $x_{i m}-x_{j m}$. The face points for each face image are converted to a binary mask and all images are resized to the size of the filter in the network. Weights are applied to all pixels in an image based on their distances from the face point mask, with greater weight given to closer pixels. Several distance measures were considered but better recognition was obtained from the Euclidean distance $\left(L^{2}\right)$ with a linear weight function that is multiplied elementwise with the input face image (Equation 2).

$$
w\left(P_{m}^{n}, F_{k}^{n}\right)=L^{2}\left(P_{m}^{n}, F_{k}^{n}\right)
$$

where $L^{2}$ is the Euclidean distance between face point $P_{m}^{n}$ and image $F_{k}^{n}, n$ corresponds to the pixel location. Pixels will have proportionally lower weights the farther they are from the corresponding facial point. Facial landmarks were incorporated into the stem block of the network as, in the Squeeze block, the resulting feature size becomes too small to calculate a binary face point mask filter. Incorporating the face points replaces the input layer of the 
original SqueezeNet [28] with an elementwise multiplication of the weight function $w$ and input layer $i_{L}$ :

$$
f^{\prime}\left\{o_{L}=i_{L} \times w\left(P_{m}^{n}, F_{k}^{n}\right)\right\}
$$

where $i_{L}$ and $o_{L}$ are the input and output of the $L-t h$ layer, and $f^{\prime}$ is the activation function.

\subsection{D SqueezExpNet (3SqEN)}

A 3D version of the SqueezExpNet architecture, which is slightly shallower than the original baseline SqueezeNet architecture, is the result of investigating variations of the Squeeze module and achieved better recognition rates over several datasets. Figure 1 shows the structure of 3D SqueezExpNet. The input size is $16 \times 3 \times 224 \times 224$ (16 the tensor size, 3 color channels, $224 \times 224$ facial image size). There are two parallel input lines to the model, one for geometry and one for texture feature maps. In "stem", these consist of two convolution layers followed by a 2D pooling layer. The geometry-based convolution layer has a lesser depth $(16 \times 111 \times$ $111 \times 64)$ than the texture-based convolutional layer $(16 \times 109 \times 109 \times 96)$. Squeeze Blocks A and B follow "stem" and consist of squeeze and expand Pooling, Dropout and convolution layers. All convolution layers are followed by a ReLU activation function to avoid the vanishing gradient problem.

As explained earlier, to capture the geometry along with the texture of the resulted feature map from $3 \mathrm{SqEN}$ and these relations into account of classifying the facial expression in the softmax layer, we used recurrent input layers (as shown in Figure 1, classifier block). The feature maps from the Squeeze Blocks enter a classifier unit after a "pool2D layer" of sizes 128 and 256. Then a recurrent input classifier concatenates the dual-stage feature maps. The traditional one-to-one type recurrent block is used as $T_{x}=T_{y}=1$ as in Equation 4 using three linear layers and a softmax $(\sigma)$ layer.

$$
\begin{gathered}
l_{1 n}=W_{l_{1}} x_{n}+U_{l_{1}} y_{n-1}+b_{l_{1}}, \\
y_{n}=\sigma_{l_{2}}\left(W_{l_{2}} l_{1 n}+b_{l_{2}}\right)
\end{gathered}
$$

where $x, y$ as input and output, $n$ is the number if iteration during the forward and backward propagation, $W, U$ and $b$ are parameter and vector. $U_{l_{1}}$ is initialized by zero value during the training process and then taken as a state layer because it has recurrent connections themselves. For the classification of still images, 128 hidden units for recurrent input were effective for facial expression recognition. A recurrent input stack captures the trend in spatial and geometric changes in the classifier, while a recurrent input classifier manages problems with gradients to 
improve the accuracy. In the final stage, the normalized exponential function is used in softmax layer $\sigma: \mathbb{R}^{C} \rightarrow \mathbb{R}^{C}$ to have a probability distribution over predicted output expression class (Equation 5).

$$
\sigma_{l_{2}}=\frac{e^{l_{2}}}{\sum_{j=1}^{C} e^{l_{2 j}}} \text { for } i=(1, \ldots, j) \in \mathbb{R}^{C}
$$

where $C$ is the total number of expression classes.

The proposed network was implemented using a combination of Pytorch and TensorFlow libraries on two Nvidia GTX1080 GPUs. In the training phase, a multi margin loss function was used with accuracy as the evaluation metric.

\section{Experiments and Discussion}

\subsection{Datasets}

This method is designed for still images of facial expressions and is evaluated on the CFEE [12], RaFD [13], and RAF-DB [14] databases.

4.1.1. Compound Facial Expressions of Emotions Database: CFEE has 5040 images from 230 subjects. There are six basic emotional expressions and neutral. As physiologists believe that the human system uses more than basic facial expressions, the database contains fifteen compound emotion categories. For example, the happily surprised expression is activation of happiness and surprised concurrently. The images are equally distributed among the basic and compound expressions for twenty-two classes (Figure 3). 

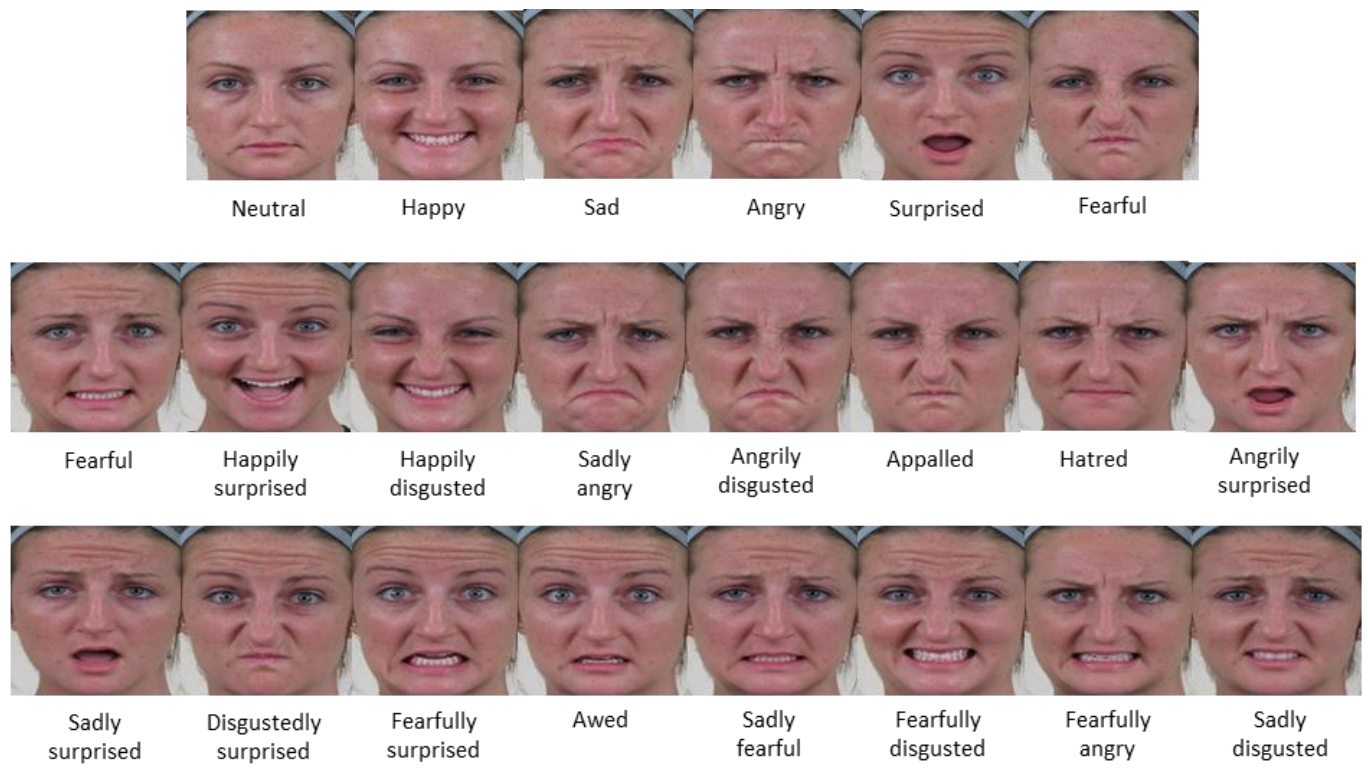

Figure 3 CFEE database with neutral, six basic and fifteen compound expressions.

4.1.2. Radboud Faces Database: RaFD is set of 67 identities displaying eight expressions including Moroccan Dutch males, Caucasian children, boys and girls both. There are 8010 images with 8 emotional expressions, happy, angry, sad, contemptuous, disgusted, fearful, surprised and neutral collected with the FACS (Facial Action Coding System) coder. To generalize to real world scenarios each expression is collected in three gaze directions looking left, front and right without changing head orientation and taken from five different camera angles simultaneously. The frontal pose is taken as the reference at zero degrees (Figure 4). The images are equally balanced per class.

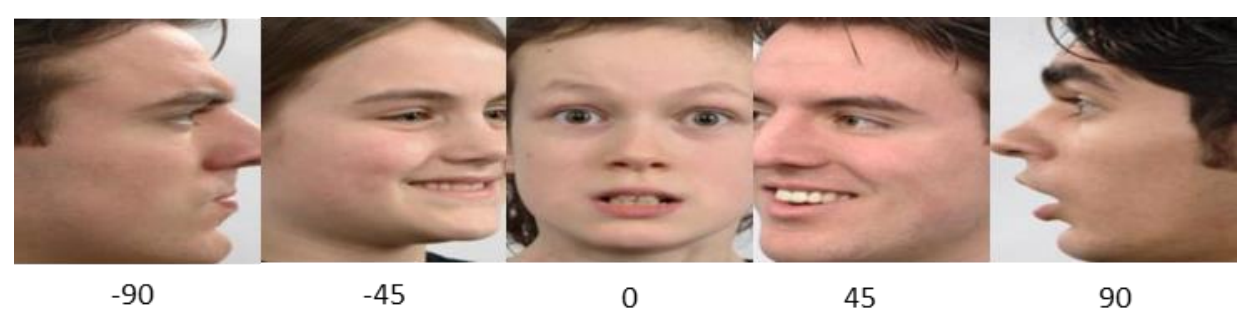

Figure 4 RaFD sample facial expressions at different camera angles.

4.1.3. Real-world Affective Faces Database: RAF-DB contains 30,681 facial images with basic and compound expressions annotated by 40 trained human coders. This dataset is composed of images collected from social media and is highly variable in terms of gender, age, ethnicity, head poses and occlusions. The subjects are up to 70 years old with $52 \%$ female, $43 \%$ male and $5 \%$ of unclear gender. $15 \%$ are of Asian, $8 \%$ of African-American, and $77 \%$ of Caucasian ethnicity. There are six basic expressions and the compound facial expressions 
happily surprised, happily disgusted, sadly fearful, sadly angry, sadly surprised, sadly disgusted, fearfully angry, fearfully surprised, angrily surprised, angrily disgusted, disgustedly surprised. The compound category contains 3162 training samples and 792 testing samples after alignment. Sample images are shown in Figure 5 In the experiment described here, 23,727 basic emotion images were used as training data and 3,000 images as test data.

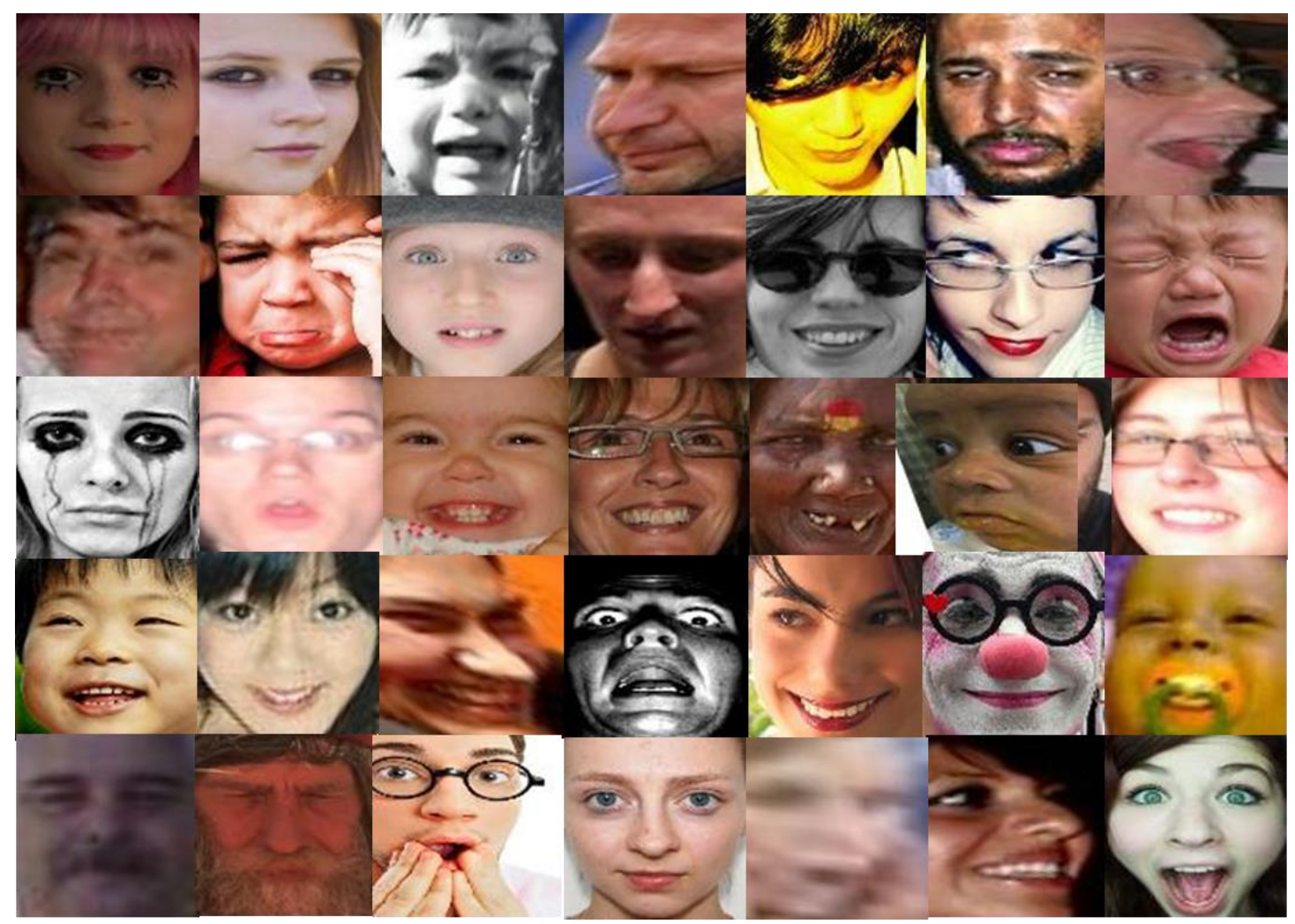

Figure 5 Real world images from the RAF-DB facial expression database.

\subsection{Results}

After detection of faces, 51 facial points are extracted by a 3D face alignment network based on a residual block method [36]. Detected faces are resized to 224 x 224 pixels. Larger image sizes will enable a deeper network with more abstract features. The network has the settings shown in Figure 1 and was trained separately for each of the three datasets. Accuracy was evaluated over a range of training parameters and the best results are reported. Two different sets of experiments were conducted to evaluate the expression recognition task and crossdataset performance.

\subsubsection{Training Parameters}


The network is trained using a multi-margin loss $\left(\mathcal{L}_{m m l}\right)$ optimization function, on a multi-class classification margin loss between the input, $x$, batch tensor and the output, $y$, tensor of a target class. For individual batch samples, the loss function is defined by Equation 6, with losses for every batch averaged over each loss element.

$$
\mathcal{L}_{m m l}(x, y)=\frac{\sum_{i=1}^{n} \max \left(0, w[y] *(\operatorname{margin}-x[y]+x[i])^{2}\right.}{x \cdot \operatorname{size}(0)}
$$

where, margin has a value of 1 and $w$ is the non-equal weighting of the classes as the RaFD and RAF-DB datasets contain unbalanced numbers of samples over classes. Weight decay, where weights are multiplied by a factor slightly less than 1 after each update prevents the weights from growing too large. This configurable hyperparameter, as used in training typically ranged from 0.0 to 1.0. The backpropagation is done at each epoch in batch. For every batch step $n$, the derivative of the loss $\mathcal{L}_{m m l}$ with respect to the weight $w$ could be expressed as in equation 7.

$$
\frac{\partial \mathcal{L}_{m m l}^{n}}{\partial w}=\left.\sum_{i=1}^{n} \frac{\partial \mathcal{L}_{m m l}^{n}}{\partial w}\right|_{i}
$$

Storing the previous $w$ is insufficient for the next best weight computation during the forward and backpropagation. An adaptive learning rate method [38] (ADADELTA) was used to optimize the network during the training process. This dynamically changes the learning rate and is robust to noisy gradients, which reduces computational load. A multi-step learning rate was incorporated to decay the learning rate when the number of epochs reach a certain level and reach the milestone i.e., 85, 120. The ADADELTA decaying process accumulates as an exponential average of the squared gradients $\left(g^{2}\right)$. Assume at epoch $n$ the running average is $S\left(g^{2}\right)_{n}$ then we compute as in equation 8 .

$$
S\left(g^{2}\right)_{n}=\rho S\left(g^{2}\right)_{n-1}+(1-\rho) g^{2}
$$

where $\rho$ is the decaying constant. Running average mean of the squared gradient is updates the previous squared gradient as in equation 9.

$$
\operatorname{RMS}(g)_{n}=\sqrt{S\left(g^{2}\right)_{n}}
$$

Then the output parameter accumulates the current and previous gradients as in equation 10 .

$$
\Delta g_{n}=-\frac{1 / R M S(g)_{n-1}}{R M S(g)_{n}}(g)_{n}
$$


(a) CFEE
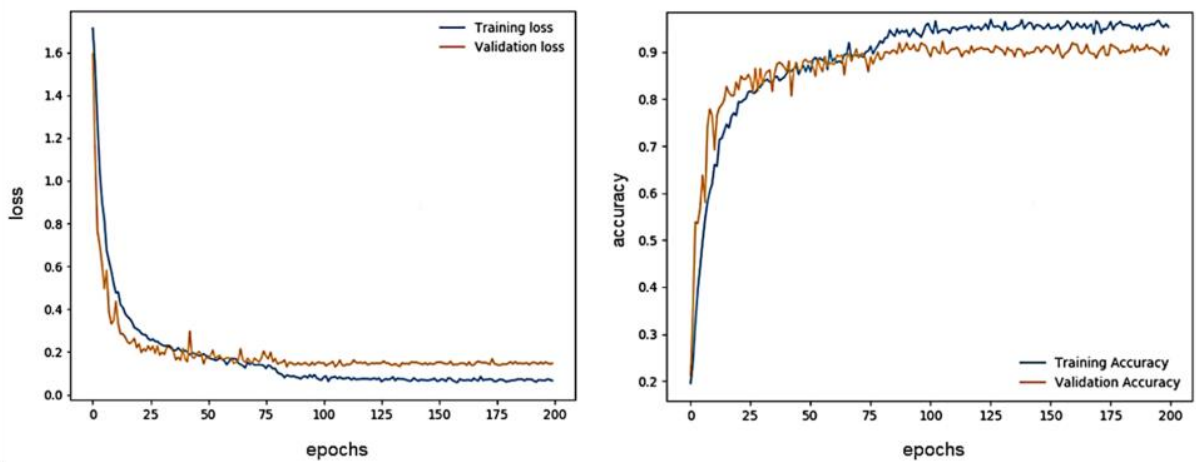

(b) RaFD
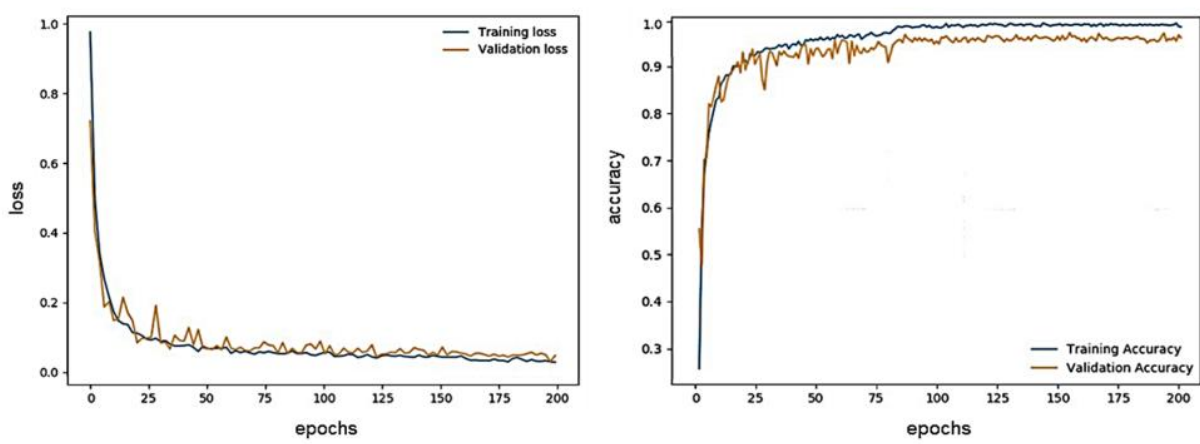

(c) RAF-DB
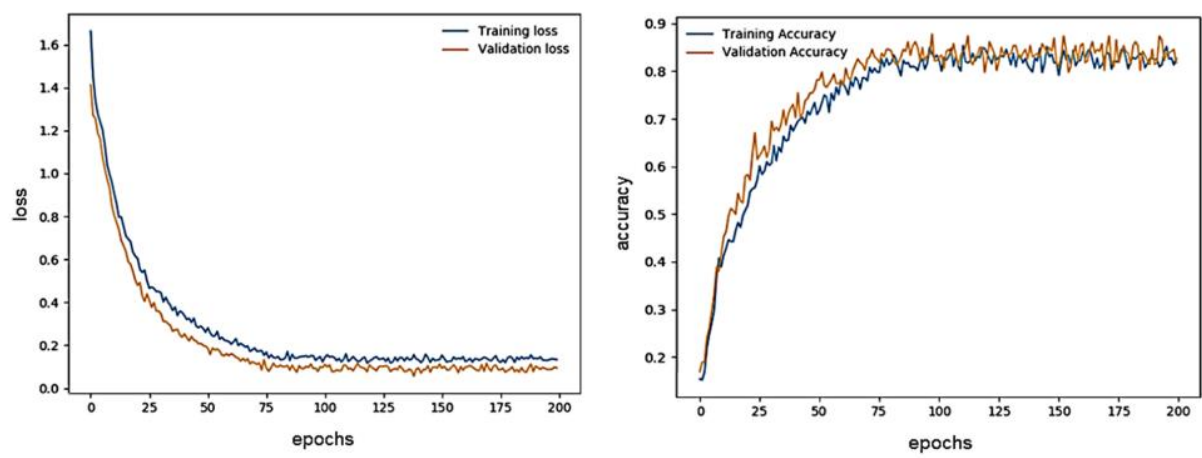

Figure 6 Convergence of loss and accuracy for training and validation in the (a) CFEE, (b) RaFD and (c) RAFDB facial expression databases.

Table 1 Recognition rate in (\%) for state-of-art methods and SqueezExpNet.

\begin{tabular}{|c|c|c|c|}
\hline \multirow[t]{2}{*}{ CNN Models } & \multicolumn{3}{|c|}{ Accuracy } \\
\hline & CFEE & RaFD & RAF-DB \\
\hline AlexNet [25] & 66.36 & 80.49 & 70.32 \\
\hline ResNet [27] & 85.14 & 82.75 & 52.40 \\
\hline RAN [39] & 56.47 & 83.45 & 51.01 \\
\hline JFDNN [40] & 71.96 & 79.86 & 63.71 \\
\hline SqueezeNet [28] & 89.19 & 90.63 & 61.91 \\
\hline SqueezExpNet ${ }^{\star}$ & $91.11 \pm 1.84$ & $95.23 \pm 1.19$ & $71.52 \pm 2.38$ \\
\hline SqueezExpNet ${ }^{\dagger}$ & $92.63 \pm 2.91$ & $96.30 \pm 1.89$ & $74.11 \pm 1.01$ \\
\hline
\end{tabular}


$\begin{array}{llll}\text { SqueezExpNet } & 93.85 \pm 1.64 & 97.12 \pm 1.58 & \mathbf{8 0 . 6 5} \pm 1.43\end{array}$

$\star$ The architecture without $3 \mathrm{D}$ face points.

$\dagger$ The accuracy directly output by Squeeze Block A \& B using linear layer

Optimizing the gradients in such way makes the training process robust to large sudden gradients and reducing the effective learning rate at current epoch. The numerator term in equation 8 acts as acceleration term by accumulating the previous gradient term over the current gradient. For keeping the similar standard, we had used 200 number of epochs during the training process of network. They convergence of loss and accuracy curves over are shown in Figure 6 for facial expression datasets on dual-stage SqueezExpNet architecture.

\subsubsection{Expression recognition task}

Each database was split into training, validation, and testing sets in a subject independent manner. The detail specification of each layer is described in Figure 1. Various kernel filter sizes, strides, and activations have been investigated and the one that had the best performance is presented in this paper. The recognition rates achieved on each database and the comparison of SqueezExpNet with published results for state-of-the-art methods are shown in Table 1. Results from SqueezExpNet without the face point mask element wise multiplication unit (it is replaced with a simple connection between the previous and next layer units), its " $2 \mathrm{D}$ " form, are also shown. Furthermore, we also had conducted experiments without using the recurrent input classifier and replaced the classifier block with the simple linear layer to concatenate the dual stages of Squeeze Block A and B. The baseline SqueezeNet doesn't contains the softmax layer for the classification, it uses the "adaptivepool2D layer". So, we also investigated the SqueezExpNet without a recurrent input-based classifier block by replacing it with a linear layer.

SqueezExpNet shows considerable improvements in the accuracy of FER (Table 1). The element-wise incorporation of 3D face points gives a greater improvement on the real-world images of the RAF-DB database than the lab posed images of the CFEE and RaFD databases. Also, the recurrent based-input classifier helps to improve the accuracy of dual-stage SqueezExpNet.

Comparing to other state-of-the-art works, our method outperforms others in RaFD and CFEE databases while achieves comparable results in RAF-DB dataset (Table 1). Most existing methods use a pre-trained network tuned for a specific database, while SqueezExpNet settings 
are the same for all databases. Due to the limited number of samples in most databases, it is challenging to train a deep network while avoiding problems like overfitting, bias and vanishing gradients. For these reasons and order to have better understanding about our proposed method, we also experimented the compound categories in facial expressions.

(a) CFEE

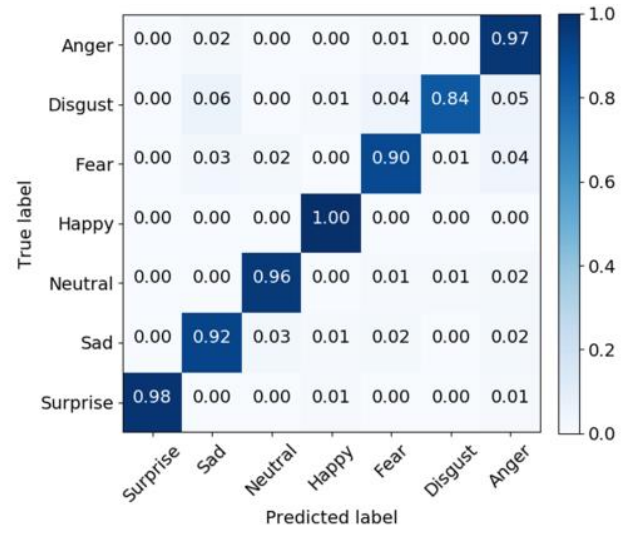

(b) RaFD

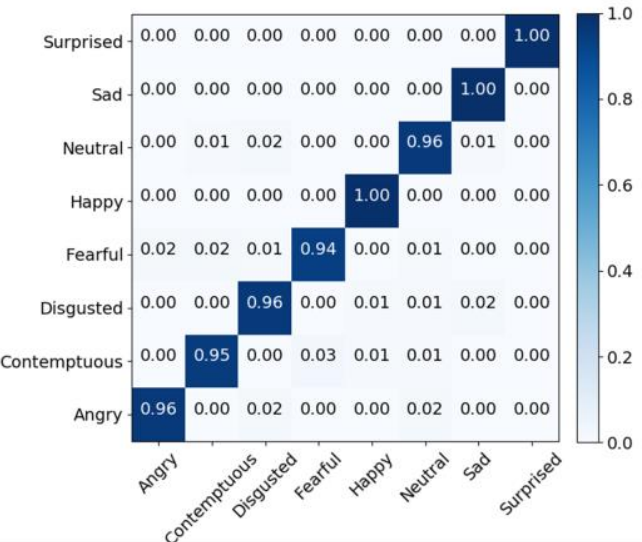

(c) RAFDB

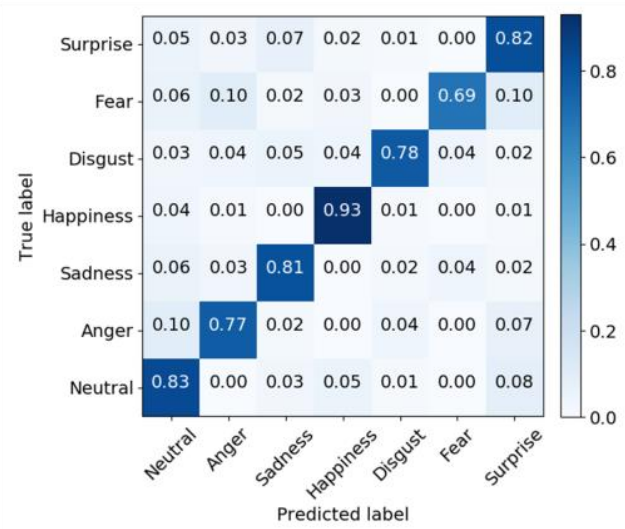

Figure 7 Confusion matrices of SqueezExpNet with face points for basic facial expressions.

Figure 7 shows the resulting confusion matrices of SqueezExpNet, with 3D face points, on different databases for basic expression recognition rates. On CFEE (Figure 7a), a higher recognition rate is achieved for the happy expression and disgust is confused with the sad $(6 \%)$ and fear (4\%) expressions. On RaFD (Figure 7b), a very high recognition rate is achieved, with surprise, sad and happy higher than the others. The highest level of confusion is between contemptuous and fearful due to the resemblance in these expressions in a side posed face. On RAFDB (Figure 7c), the highest and lowest recognition accuracies are achieved by happiness and fear, respectively. This level of confusion is reasonable considering that RAF-DB is a highly imbalanced database and this has biased the network towards the disgust and fear expression categories. Some of the real-world images are highly occluded with, for example, sunglasses or makeup (Figure 5). Despite the low number of images in disgust and fear in RAF- 
DB database, the most difficult expressions to identify among all the databases, SqueezExpNet is able to achieve better recognition rates relative to other methods. Happy and surprise are the only expressions that were more recognized across all the databases and their misclassification rate is lower than rest of the expression classes (Figure 7a-c).

Table 2 Confusion matrix for compound categories when using SqueezExpNet on the CFEE database. a: Happily surprised, b: Happily disgusted, c: Sadly fearful, d: Sadly angry, e: Sadly surprised, f: Sadly disgusted, g: Fearfully angry, h: Fearfully surprised, i: Fearfully disgusted, j: Angrily surprised, k: Angrily disgusted, l: Disgustedly surprised, m: Appalled, n: Hatred, o: Awed.

\begin{tabular}{llllllllllllllll}
\hline$\%$ & $\mathbf{a}$ & $\mathbf{b}$ & $\mathbf{c}$ & $\mathbf{d}$ & $\mathbf{e}$ & $\mathbf{f}$ & $\mathbf{g}$ & $\mathbf{h}$ & $\mathbf{i}$ & $\mathbf{j}$ & $\mathbf{k}$ & $\mathbf{l}$ & $\mathbf{m}$ & $\mathbf{n}$ & $\mathbf{o}$ \\
\hline $\mathbf{a}$ & $\mathbf{8 7 . 5}$ & 0.0 & 2.1 & 0.0 & 4.2 & 0.0 & 0.0 & 0.0 & 6.3 & 0.0 & 0.0 & 0.0 & 0.0 & 0.0 & 0.0 \\
$\mathbf{b}$ & 0.0 & $\mathbf{1 0 0 . 0}$ & 0.0 & 0.0 & 0.0 & 0.0 & 0.0 & 0.0 & 0.0 & 0.0 & 0.0 & 0.0 & 0.0 & 0.0 & 0.0 \\
$\mathbf{c}$ & 0.0 & 0.0 & $\mathbf{8 3 . 3}$ & 2.1 & 0.0 & 0.0 & 2.1 & 0.0 & 0.0 & 0.0 & 6.3 & 0.0 & 4.2 & 0.0 & 2.1 \\
$\mathbf{d}$ & 2.1 & 0.0 & 2.1 & $\mathbf{7 7 . 1}$ & 2.1 & 2.1 & 2.1 & 12.5 & 0.0 & 0.0 & 0.0 & 0.0 & 0.0 & 0.0 & 0.0 \\
$\mathbf{e}$ & 0.0 & 2.1 & 0.0 & 2.1 & $\mathbf{8 7 . 5}$ & 2.1 & 2.1 & 2.1 & 0.0 & 0.0 & 0.0 & 0.0 & 0.0 & 2.1 & 0.0 \\
$\mathbf{f}$ & 2.1 & 0.0 & 0.0 & 0.0 & 2.1 & $\mathbf{6 8 . 8}$ & 0.0 & 2.1 & 0.0 & 6.3 & 0.0 & 4.2 & 0.0 & 14.6 & 0.0 \\
$\mathbf{g}$ & 2.1 & 0.0 & 0.0 & 0.0 & 2.1 & 4.2 & $\mathbf{7 5 . 0}$ & 0.0 & 12.5 & 0.0 & 0.0 & 4.2 & 0.0 & 0.0 & 0.0 \\
$\mathbf{h}$ & 4.2 & 0.0 & 2.1 & 2.1 & 0.0 & 0.0 & 0.0 & $\mathbf{8 1 . 3}$ & 0.0 & 2.1 & 0.0 & 0.0 & 4.2 & 0.0 & 4.2 \\
$\mathbf{i}$ & 2.1 & 2.1 & 0.0 & 4.2 & 0.0 & 0.0 & 0.0 & 0.0 & $\mathbf{8 3 . 3}$ & 0.0 & 0.0 & 0.0 & 6.3 & 0.0 & 2.1 \\
$\mathbf{j}$ & 0.0 & 0.0 & 0.0 & 0.0 & 0.0 & 2.1 & 0.0 & 0.0 & 0.0 & $\mathbf{9 7 . 9}$ & 0.0 & 0.0 & 0.0 & 0.0 & 0.0 \\
$\mathbf{k}$ & 0.0 & 2.1 & 0.0 & 0.0 & 2.1 & 0.0 & 0.0 & 0.0 & 0.0 & 0.0 & $\mathbf{9 3 . 8}$ & 0.0 & 2.1 & 0.0 & 0.0 \\
$\mathbf{l}$ & 0.0 & 0.0 & 0.0 & 2.1 & 0.0 & 0.0 & 2.1 & 2.1 & 0.0 & 0.0 & 0.0 & $\mathbf{9 3 . 8}$ & 0.0 & 0.0 & 0.0 \\
$\mathbf{m}$ & 0.0 & 0.0 & 0.0 & 4.2 & 0.0 & 0.0 & 10.4 & 0.0 & 0.0 & 0.0 & 2.1 & 2.1 & $\mathbf{7 2 . 9}$ & 0.0 & 0.0 \\
$\mathbf{n}$ & 0.0 & 0.0 & 4.2 & 2.1 & 2.1 & 2.1 & 2.1 & 0.0 & 0.0 & 0.0 & 0.0 & 2.1 & 0.0 & $\mathbf{8 5 . 4}$ & 0.0 \\
$\mathbf{0}$ & 0.0 & 2.1 & 2.1 & 0.0 & 0.0 & 0.0 & 2.1 & 6.3 & 4.2 & 2.1 & 0.0 & 0.0 & 0.0 & 0.0 & $\mathbf{8 1 . 3}$ \\
\hline
\end{tabular}

\subsubsection{Compound Expressions}

To classify compound expressions the CFEE and RAF-DB datasets were used with the basic expression categories removed for some experiments. The mean diagonal values in the confusion matrix of the 15 classes for the CFEE dataset show the best classification is achieved for the happily disgusted and angrily surprised expressions (Table 2) with the least accuracy (72.9\%) achieved for the appalled expression For an overall accuracy of $84.58 \%$ while using the compound expressions. The RAF-DB dataset compound expressions were also considered and SqueezExpNet achieved an average accuracy of $65.26 \%$ over the 11 accessible classes (Table 3). Performance decreases substantially when more real-world expressions are included and are significantly lower than results for basic expressions. Compound expressions are more 
difficult to detect, and more robust methods need to be developed to solve this problem. Compound expressions are less represented in the RAF-DB dataset with only 3,954 images in total. Compound expressions from real-world images are another major challenge.

Table 3 Confusion matrix for compound categories when using SqueezExpNet for RAFDB database. a: Happily Surprised, b: Happily Disgusted, c: Sadly Fearful, d: Sadly Angry, e: Sadly Surprised, f: Sadly Disgusted, g: Fearfully Angry, h: Fearfully Surprised, i: Angrily Surprised, j: Angrily Disgusted, k: Disgustedly Surprised.

\begin{tabular}{llllllllllll}
\hline \% & $\mathbf{a}$ & $\mathbf{b}$ & $\mathbf{c}$ & $\mathbf{d}$ & $\mathbf{e}$ & $\mathbf{f}$ & $\mathbf{g}$ & $\mathbf{h}$ & $\mathbf{i}$ & $\mathbf{j}$ & $\mathbf{k}$ \\
\hline $\mathbf{a}$ & $\mathbf{7 1 . 1}$ & 3.3 & 0.8 & 0 & 0 & 0.1 & 9.4 & 1.5 & 4 & 4.5 & 5.3 \\
$\mathbf{b}$ & 11.2 & $\mathbf{6 9 . 5}$ & 0 & 1.3 & 2.1 & 0 & 5.5 & 6.9 & 0.7 & 1.9 & 0.9 \\
$\mathbf{c}$ & 3.5 & 1.2 & $\mathbf{4 2 . 9}$ & 23.8 & 0 & 2.1 & 9.8 & 0.8 & 4.5 & 7.8 & 3.6 \\
$\mathbf{d}$ & 0 & 7.4 & 4.4 & $\mathbf{6 3 . 8}$ & 8.6 & 1.1 & 0 & 4.8 & 1.9 & 8 & 0 \\
$\mathbf{e}$ & 0 & 2 & 2.8 & 1.7 & $\mathbf{7 3 . 5}$ & 0 & 6.4 & 1.1 & 0.4 & 9.8 & 2.3 \\
$\mathbf{f}$ & 4.1 & 0 & 0 & 6.8 & 8.9 & $\mathbf{6 1 . 4}$ & 15.4 & 3.2 & 0 & 0.2 & 0 \\
$\mathbf{g}$ & 5.6 & 0.8 & 3.2 & 1.1 & 6.3 & 0 & $\mathbf{6 5 . 8}$ & 1 & 15.1 & 1.1 & 0 \\
$\mathbf{h}$ & 21.1 & 0.5 & 0 & 8.7 & 0 & 6.1 & 0 & $\mathbf{6 0 . 7}$ & 0 & 2.9 & 0 \\
$\mathbf{i}$ & 9.8 & 0 & 6.1 & 0 & 0 & 5.5 & 2.5 & 10.8 & $\mathbf{6 1 . 1}$ & 4.2 & 0 \\
$\mathbf{j}$ & 1.1 & 3.5 & 0 & 1.4 & 3.6 & 1.1 & 4.9 & 0.8 & 4.5 & $\mathbf{7 7 . 2}$ & 1.9 \\
$\mathbf{k}$ & 1.7 & 0 & 0.8 & 1.1 & 1.6 & 0.4 & 6 & 7.9 & 1.6 & 8.1 & $\mathbf{7 0 . 8}$ \\
\hline
\end{tabular}

DCNN models are applied to many visual recognition tasks and have performed remarkably well using large sets of training samples. However, on FER tasks, DCNN models are very easy to be overfitted due to a lack of insufficient training data. SqueezExpNet was compared with DCNN models using compound expressions (Table 4). Most frameworks employed for FER, such as RAN [39] and JFDNN [40], are based on pre-trained models. AlexNet [42], ResNet [27] and SqueezeNet [28] were initially designed for ImageNet, a database of thousands of objects that is not well suited to expression characteristics. Therefore, SqueezExpNet was trained on a different dataset like controlled, posed and uncontrolled facial expression data. The performance of SqueezExpNet improved by $2.51 \%$ and $1.82 \%$ for the CFEE and RAF-DB datasets by adding basic expression classes to the compound expressions.

Table 4 Expression performance of different deep neural networks on basic and compound expression categories. The metric is the mean diagonal value of the confusion matrix.

\begin{tabular}{|c|c|c|c|c|c|c|c|c|c|c|}
\hline & & \multicolumn{8}{|c|}{ Basic } & \multirow{2}{*}{$\begin{array}{c}\text { Compound } \\
\text { Accuracy }\end{array}$} \\
\hline & & Anger & Disgust & Fear & Happy & Neutral & $\mathrm{Sad}$ & Surprise & Average $\dagger$ & \\
\hline CFEE & AlexNet [25] & 61.52 & 50.94 & 44.64 & 67.19 & 51.47 & 70.2 & 67.11 & 59.01 & 63.26 \\
\hline
\end{tabular}


Submitted for expected publication in JAIHC

\begin{tabular}{|c|c|c|c|c|c|c|c|c|c|c|}
\hline & ResNet [27] & 75.89 & 78.19 & 69.73 & 79.19 & 79.65 & 80.64 & 84.67 & 78.28 & 58.22 \\
\hline & RAN [39] & 45.75 & 60.91 & 16.06 & 57.71 & 61.91 & 47.39 & 67.08 & 50.97 & 72.74 \\
\hline & JFDNN [40] & 56.04 & 59.52 & 64.48 & 79.64 & 44.97 & 65.47 & 77.9 & 64 & 59.5 \\
\hline & SqueezeNet [28] & 77.62 & 79.48 & 80.52 & 89.93 & 80.25 & 76.59 & 87.92 & 81.76 & 78.69 \\
\hline & SqueezExpNet & 89.54 & 76.63 & 82.17 & 92.5 & 88.7 & 84.13 & 90.51 & 86.31 & 89.09 \\
\hline \multirow{6}{*}{$\begin{array}{c}\text { RAF- } \\
\text { DB }\end{array}$} & AlexNet [25] & 48.14 & 45.58 & 70.6 & 71.94 & 72.81 & 57.37 & 77.4 & 63.41 & 32.12 \\
\hline & ResNet [27] & 36.39 & 45.4 & 50.48 & 53.59 & 41.15 & 35.79 & 54.32 & 45.3 & 29.33 \\
\hline & RAN [39] & 33.6 & 26.66 & 47.16 & 45.93 & 59.67 & 41.05 & 51.8 & 43.7 & 40.19 \\
\hline & JFDNN [40] & 64.58 & 55.67 & 47.69 & 59.83 & 44.64 & 48.1 & 69.63 & 55.73 & 41.61 \\
\hline & SqueezeNet [28] & 45.52 & 37.26 & 62.24 & 69.42 & 52.38 & 38.61 & 70.99 & 53.77 & 27.55 \\
\hline & SqueezExpNet & 69.48 & 68.61 & 47.53 & 75.28 & 75.62 & 73.82 & 74.6 & 69.28 & 71.10 \\
\hline
\end{tabular}

${ }^{\dagger}$ The mean diagonal value of confusion matrix

Table 4 shows the performance of expression recognition of different DCNN's on CFEE and RAF-DB datasets. The metric is the mean diagonal value of the confusion matrix for the basic and compound expressions. Form the results, we have the following observations. First, DCNN's, which achieve reasonable results for large scale images recognition settings, such as the AlexNet network and ResNet, are not efficient for facial expression recognition. Second, the deep features from SqueezeNet type of architecture outperform on the unlearned features used in the baseline system by significant margin. This also indicates that the SqueezExpNet deep learning architecture is more robust and applicable for both basic and compound expression classification.

\subsection{Cross Dataset Study}

A cross dataset study, where one dataset of images is used for training and another for testing, was performed to explore specific differences between the lab-controlled faces guided by psychologists and real-word expressions using the same architecture as described in Figure 1. The same network architecture as expression recognition task was used for this task. Table 5 shows the performance of facial expression on each database in the cross-dataset study. The Fscore is defined as $(2 *$ Recall $*$ Precision $) /($ Recall + Precision $)$.

Table 5 Cross dataset performance in CFEE, RaFD and RAF-DB.

\begin{tabular}{|c|c|c|c|c|c|c|}
\hline Train & CFEE & & RaFD & & RAF-I & \\
\hline Test & RaFD & RAF-DB & CFEE & RAF-DB & CFEE & RaFD \\
\hline Accuracy & 62.71 & 40.51 & 51.54 & 41.93 & 54.76 & 67.52 \\
\hline F-score & 50.72 & 37.63 & 50.80 & 38.47 & 52.83 & 62.08 \\
\hline
\end{tabular}

\section{CONCLUSION}


In this work, we presented a deep neural network for facial expression recognition, 3D SqueezExpNet (3SqEN) which extends SqueezeNet [28] for expression classification. The addition of a 3D face points mask gives volume to the feature map and extracts spatial relations of facial images and allows differentiation between important facial components and other parts of the. The feature unit is followed by a recurrent base classifier to group the geometrical and spatial stages. The face point masks are multiplied element wise with the tensor in the stem and squeeze modules which also reduce the block of squeeze and expand layers in the traditional SqueezeNet.

3D SqueezExpNet was evaluated for basic facial expression recognition and in a cross database study. Three facial expression databases (CFEE, RaFD and RAF-DB) that range from lab posed to uncontrolled basic and compound images were used for evaluation. In both tasks 3D SqueezExpNet outperformed many state-of-the-art methods and offers a general solution for FER.

\section{ACKWNOWLEDGMENT}

This work is supported by City University of Hong Kong (Project 9610034) and the Hong Kong Institute for Data Science.

\section{References}

[1] A. Mehrabian, S.R. Ferris, Inference of attitudes from nonverbal communication in two channels, J. Consult. Psychol. (1967). doi:10.1037/h0024648.

[2] P. Ekman, W. V Friesen, Manual for the facial action coding system, Consult. Psychol. (1978). doi:10.1029/1999JB900207.

[3] S. Li, W. Deng, Blended Emotion in-the-Wild: Multi-label Facial Expression Recognition Using Crowdsourced Annotations and Deep Locality Feature Learning, Int. J. Comput. Vis. (2019). doi:10.1007/s11263-018-1131-1.

[4] W. Gu, C. Xiang, Y. V. Venkatesh, D. Huang, H. Lin, Facial expression recognition using radial encoding of local Gabor features and classifier synthesis, Pattern Recognit. (2012). doi:10.1016/j.patcog.2011.05.006.

[5] Y. Guo, G. Zhao, M. Pietikäinen, Discriminative features for texture description, Pattern Recognit. (2012). doi:10.1016/j.patcog.2012.04.003. 
[6] S. Khan, G. Xu, R. Chan, H. Yan, An online spatio-temporal tensor learning model for visual tracking and its applications to facial expression recognition, Expert Syst. Appl. (2017). doi:10.1016/j.eswa.2017.08.039.

[7] M. Nawaz, H. Yan, Saliency detection via multiple-morphological and superpixel based fast fuzzy C-mean clustering network, Expert Syst. Appl. (2020). doi:10.1016/j.eswa.2020.113654.

[8] M. Zhang, S. Khan, H. Yan, Deep eigen-filters for face recognition: Feature representation via unsupervised multi-structure filter learning, Pattern Recognit. (2020). doi:10.1016/j.patcog.2019.107176.

[9] R. Qureshi, M. Uzair, K. Khurshid, H. Yan, Hyperspectral document image processing: Applications, challenges and future prospects, Pattern Recognit. (2019). doi:10.1016/j.patcog.2019.01.026.

[10] T.M. Khan, M. Alhussein, K. Aurangzeb, M. Arsalan, S.S. Naqvi, S.J. Nawaz, Residual Connection-Based Encoder Decoder Network (RCED-Net) for Retinal Vessel Segmentation, IEEE Access. (2020). doi:10.1109/ACCESS.2020.3008899.

[11] A.T. Lopes, E. de Aguiar, A.F. De Souza, T. Oliveira-Santos, Facial expression recognition with Convolutional Neural Networks: Coping with few data and the training sample order, Pattern Recognit. (2017). doi:10.1016/j.patcog.2016.07.026.

[12] S. Du, Y. Tao, A.M. Martinez, Compound facial expressions of emotion, Proc. Natl. Acad. Sci. U. S. A. (2014). doi:10.1073/pnas.1322355111.

[13] O. Langner, R. Dotsch, G. Bijlstra, D.H.J. Wigboldus, S.T. Hawk, A. van Knippenberg, Presentation and validation of the radboud faces database, Cogn. Emot. (2010). doi:10.1080/02699930903485076.

[14] S. Li, W. Deng, Reliable crowdsourcing and deep locality-preserving learning for unconstrained facial expression recognition, IEEE Trans. Image Process. (2019). doi:10.1109/TIP.2018.2868382.

[15] S. Li, W. Deng, Deep Facial Expression Recognition: A Survey, IEEE Trans. Affect. Comput. (2020). doi:10.1109/TAFFC.2020.2981446.

[16] Y. Lei, M. Bennamoun, M. Hayat, Y. Guo, An efficient 3D face recognition approach using local geometrical signatures, Pattern Recognit. (2014). doi:10.1016/j.patcog.2013.07.018. 
[17] N. Dalal, B. Triggs, Histograms of oriented gradients for human detection, in: Proc. 2005 IEEE Comput. Soc. Conf. Comput. Vis. Pattern Recognition, CVPR 2005, 2005. doi:10.1109/CVPR.2005.177.

[18] C. Shan, S. Gong, P.W. McOwan, Facial expression recognition based on Local Binary Patterns: A comprehensive study, Image Vis. Comput. (2009). doi:10.1016/j.imavis.2008.08.005.

[19] C. Liu, H. Wechsler, Gabor feature based classification using the enhanced Fisher linear discriminant model for face recognition, IEEE Trans. Image Process. (2002). doi:10.1109/TIP.2002.999679.

[20] A.R. Shahid, S. Khan, H. Yan, Human expression recognition using facial shape based Fourier descriptors fusion, in: 2020. doi:10.1117/12.2557450.

[21] K.M. Lam, H. Yan, An analytic-to-holistic approach for face recognition based on a single frontal view, IEEE Trans. Pattern Anal. Mach. Intell. (1998). doi:10.1109/34.689299.

[22] I. Kotsia, I. Pitas, Facial expression recognition in image sequences using geometric deformation features and support vector machines, IEEE Trans. Image Process. (2007). doi:10.1109/TIP.2006.884954.

[23] S. Khan, L. Chen, H. Yan, Co-clustering to reveal salient facial features for expression recognition, IEEE Trans. Affect. Comput. (2017). doi:10.1109/TAFFC.2017.2780838.

[24] Z.U. Rehman, S.S. Naqvi, T.M. Khan, M.A. Khan, T. Bashir, Fully automated multiparametric brain tumour segmentation using superpixel based classification, Expert Syst. Appl. (2019). doi:10.1016/j.eswa.2018.10.040.

[25] A. Krizhevsky, I. Sutskever, G.E. Hinton, ImageNet classification with deep convolutional neural networks, Commun. ACM. (2017). doi:10.1145/3065386.

[26] K. Simonyan, A. Zisserman, Very deep convolutional networks for large-scale image recognition, in: 3rd Int. Conf. Learn. Represent. ICLR 2015 - Conf. Track Proc., 2015.

[27] K. He, X. Zhang, S. Ren, J. Sun, Deep residual learning for image recognition, in: Proc. IEEE Comput. Soc. Conf. Comput. Vis. Pattern Recognit., 2016. doi:10.1109/CVPR.2016.90.

[28] F.N. Iandola, M.W. Moskewicz, K. Ashraf, S. Han, W.J. Dally, K. Keutzer, SqueezeNet, ArXiv. (2016). 
[29] C. Li, X. Wei, H. Yu, J. Guo, X. Tang, Y. Zhang, An Enhanced SqueezeNet Based Network for Real-Time Road-Object Segmentation, in: 2019 IEEE Symp. Ser. Comput. Intell. SSCI 2019, 2019. doi:10.1109/SSCI44817.2019.9002818.

[30] M. Längkvist, L. Karlsson, A. Loutfi, Inception-v4, Inception-ResNet and the Impact of Residual Connections on Learning, Pattern Recognit. Lett. (2014). doi:10.1016/j.patrec.2014.01.008.

[31] T. Zhang, W. Zheng, Z. Cui, Y. Zong, Y. Li, Spatial-Temporal Recurrent Neural Network for Emotion Recognition, IEEE Trans. Cybern. (2019). doi:10.1109/TCYB.2017.2788081.

[32] N. Jain, S. Kumar, A. Kumar, P. Shamsolmoali, M. Zareapoor, Hybrid deep neural networks for face emotion recognition, Pattern Recognit. Lett. (2018). doi:10.1016/j.patrec.2018.04.010.

[33] J. Gu, Z. Wang, J. Kuen, L. Ma, A. Shahroudy, B. Shuai, T. Liu, X. Wang, G. Wang, J. Cai, T. Chen, Recent advances in convolutional neural networks, Pattern Recognit. (2018). doi:10.1016/j.patcog.2017.10.013.

[34] H.J. Lee, I. Ullah, W. Wan, Y. Gao, Z. Fang, Real-Time vehicle make and model recognition with the residual squeezenet architecture, Sensors (Switzerland). (2019). doi:10.3390/s19050982.

[35] F. Ucar, D. Korkmaz, COVIDiagnosis-Net: Deep Bayes-SqueezeNet based diagnosis of the coronavirus disease 2019 (COVID-19) from X-ray images, Med. Hypotheses. (2020). doi:10.1016/j.mehy.2020.109761.

[36] A. Bulat, G. Tzimiropoulos, How Far are We from Solving the 2D \& 3D Face Alignment Problem? (and a Dataset of 230,000 3D Facial Landmarks), in: Proc. IEEE Int. Conf. Comput. Vis., 2017. doi:10.1109/ICCV.2017.116.

[37] S. Jaiswal, M. Valstar, Deep learning the dynamic appearance and shape of facial action units, in: 2016 IEEE Winter Conf. Appl. Comput. Vision, WACV 2016, 2016. doi:10.1109/WACV.2016.7477625.

[38] Zeiler, M.D., Adadelta: An Adaptive Learning Rate Method, arXiv preprint (2012). arXiv:1212.5701

[39] K. Wang, X. Peng, J. Yang, D. Meng, Y. Qiao, Region Attention Networks for Pose and Occlusion Robust Facial Expression Recognition, IEEE Trans. Image Process. (2020). doi:10.1109/TIP.2019.2956143. 
Submitted for expected publication in JAIHC

[40] H. Jung, S. Lee, J. Yim, S. Park, J. Kim, Joint fine-tuning in deep neural networks for facial expression recognition, in: Proc. IEEE Int. Conf. Comput. Vis., 2015. doi:10.1109/ICCV.2015.341. 